\title{
Water use of sorghum (Sorghum bicolor L. Moench) in response to varying planting dates evaluated under rainfed conditions
}

\author{
Sandile T Hadebe ${ }^{1 *}$, Tafadzwanashe Mabhaudhi' and Albert T Modi' \\ 'Crop Science, School of Agricultural, Earth and Environmental Sciences, University of KwaZulu-Natal, Private Bag X01, Scottsville 3209, \\ Pietermaritzburg, South Africa
}

\begin{abstract}
It is vital to understand how rainfall onset, amount and distribution between planting dates affect sorghum yield and water use, in order to aid planting date and cultivar selection. This study investigated morphological, physiological, phenological, yield and water use characteristics of different sorghum genotypes in response to different planting dates under rainfed conditions. Four genotypes (PAN8816 [hybrid], Macia [open-pollinated variety, OPV], Ujiba and IsiZulu [both landraces]) were planted on 3 planting dates (early, optimal, and late) in a split-plot design, with planting dates as the main factor. Low soil water at the optimal planting date was associated with delayed crop establishment and low final emergence. Sorghum genotypes adapted to low and irregular rainfall at the late planting date through low leaf number, canopy cover, chlorophyll content index and stomatal conductance, and hastened phenological development. This resulted in low biomass and grain yields. Landraces exhibited grain yield stability across planting dates, whilst OPV and hybrid genotypes significantly reduced grain yield in response to low water availability when planted late. Biomass and grain yield water use efficiency (WUE) were highest at optimal planting date (30.5 and $\left.9.2 \mathrm{~kg} \cdot \mathrm{ha}^{-1} \cdot \mathrm{mm}^{-1}\right)$, relative to late $\left(23.1\right.$ and $\left.8.7 \mathrm{~kg} \cdot \mathrm{ha}^{-1} \cdot \mathrm{mm}^{-1}\right)$, and early planting dates $\left(25.2\right.$ and $\left.8.3 \mathrm{~kg} \cdot \mathrm{ha}^{-1} \cdot \mathrm{mm}^{-1}\right)$. For PAN8816 and Macia, biomass and grain WUE decreased in response to low soil water content, and irregular and disproportionate rainfall experienced during the late planting date. By contrast, biomass and grain WUE for Ujiba and IsiZulu improved with decreasing rainfall. PAN8816 is recommended when planting under low soil water availability to maximize crop stand. Cultivation of Macia is recommended under optimal conditions. Ujiba and IsiZulu landraces are recommended for low rainfall areas with highly variable rainfall. Repetition or modelling of genotype responses across environments different from Ukulinga is required for thorough water use characterisation of these genotypes.
\end{abstract}

ABSTRACT

Keywords: planting dates, water use efficiency, rainfall variability, cultivar selection, landraces and improved sorghum varieties

\section{INTRODUCTION}

Sub-optimal availability of water for unrestricted plant growth and transpiration, i.e., drought, is a major limitation to agricultural production (Delmer, 2005). Rainfall can be erratic and irregular in sub-Saharan Africa (SSA) (Chauvin et al., 2012), where climate variability mainly impacts resource-poor farmers, whose livelihoods depend mainly on rainfed agriculture (Tadross et al., 2005). The inability of this group of farmers to adapt to changing or variable weather patterns makes them increasingly vulnerable and prone to repeated episodes of crop failure and food insecurity. For farmers relying on rainfed agriculture, the ability to adapt to changing weather patterns on a season-toseason basis is a prerequisite to successful crop production. There are various strategic and tactical decisions that can allow farmers to adapt to changing and variable weather patterns. On a tactical level, these include crop or cultivar choice and planting date selection.

Traditionally, farmers use the onset of the rainy season as a criterion for setting planting dates. Multiple planting dates can occur in a single rainy season, depending on the onset and longevity of rainy season, and optimal crop growing period. Successful rainfed agriculture is largely dependent upon availability of free and stored rainwater, but rainfall variability in both total amounts and seasonal distribution is a challenge

${ }^{\star}$ To whom all correspondence should be addressed.

Tel: +27 33 260-5447; Fax: +27 33 260-6094; e-mail: hadebesta@gmail.com Received: 29 September 2015; accepted in revised form: 5 January 2017
(Rockström and Barron, 2007). Inappropriate planting dates can result in crop failure, usually requiring replanting or reduced yield as a consequence of unmet water requirements. Crops adapt to diverse environments through considerable plasticity of phenology, morphology and physiology, the main determinant of which is rainfall (Udungwu and Summerfield, 1985). One of the ways of manipulating this climatic factor is adequate knowledge of optimal planting dates so as to accurately synchronize rainfall incidences with crop development (Adetayo et al., 2008).

Sorghum has been reported to have high stress tolerance, including temperature, water and salt stresses (Ejeta and Knoll, 2007). The South African Department of Agriculture and Forestry (DAFF, 2010) recommended optimal planting time for sorghum in South Africa is from early November until the end of December; with the dates falling on either side of the recommended times regarded as early and late planting, respectively. This recommendation covers all sorghum growing regions of South Africa, irrespective of climatic variation within regions. The first sowing date can be defined as the first rainfall event capable of supporting germination (Keatinge et al., 1995). Delays in the onset of rainfall, drought seasons, unpredictable periodic dry spells and lengthened rainfall season have led to a shift in traditionally recommended sorghum planting dates (Yamusa et al., 2013). Knowledge of cultivar performance under different planting dates in SSA, for mitigation and production plasticity under different planting date scenarios, is lacking. Therefore, characterising crop water use responses for sorghum as an alternative staple cereal crop, in dryland farming under different planting dates has positive implications for food security. This study investigated morphological, physiological, 
phenological, yield and water use characteristics of different sorghum genotypes in response to different planting dates under dryland production. It was hypothesised that crop water use responses of different sorghum genotypes would differ given variations in planting dates.

\section{MATERIALS AND METHODS}

\section{Plant material}

Four genotypes of sorghum were used, namely PAN8816, Macia, Ujiba and IsiZulu (landraces from KwaZulu-Natal). PAN8816, a semi-dwarf genotype, was sourced from Pannar Seeds. It is a bronze-grained, medium- to late-maturing, low-tannin hybrid renowned for leaf disease and head smut resistance. Flowering occurs approximately 71 days after sowing. Macia is an early- to medium-maturing (60-65 days to floral initiation and 115-120 days to maturity), semi-dwarf (1.3-1.5 m tall with thick stem), low-tannin open-pollinated variety. It has good drought tolerance $(250-750 \mathrm{~mm}$ rainfall range during the growing season), with stay-green characteristics extending beyond harvest. Yield potential is $3-6 \mathrm{t} \cdot \mathrm{ha}^{-1}$. Ujiba is a reddishbrown seeded, tall growing $(>1.5 \mathrm{~m})$, high-tannin landrace genotype sourced locally from smallholder farmers in Tugela Ferry $\left(28^{\circ} 44^{\prime} \mathrm{S}, 30^{\circ} 27^{\prime} \mathrm{E}\right)$. IsiZulu is a dark-brown seeded, tall growing $(>1.5 \mathrm{~m})$, high-tannin landrace genotype sourced locally from smallholder farmers at Nkandla ( $\left.28^{\circ} 50^{\prime} \mathrm{S}, 31^{\circ} 06^{\prime} \mathrm{E}\right)$. For landraces, phenological, morphological and physiological information was lacking prior to initiation of the experiment.

\section{Agronomic practices}

Soil samples were collected and analysed for fertility before land preparation. Before planting, the fallow land was mechanically ploughed, disked and rotovated. A glyphosate (Round-up) pre-emergence herbicide $(10 \mathrm{~mL}$ per $1 \mathrm{~L}$ of water), was applied to control weeds 2 weeks before planting. Smith (2006) recommends that for an expectation of $6 \mathrm{~kg} \cdot \mathrm{ha}^{-1}$ of yield, 120 $\mathrm{kg} \cdot \mathrm{ha}^{-1}$ of nitrogen should be applied. After soil analysis, a deficit of fertiliser requirements was applied using Gromor Accelerator (30 g. $\mathrm{kg}^{-1} \mathrm{~N}, 15 \mathrm{~g} \cdot \mathrm{kg}^{-1} \mathrm{P}$ and $15 \mathrm{~g} \cdot \mathrm{kg}^{-1} \mathrm{~K}$ ) a slow-releasing organic fertilizer, 14 days after sowing (DAS) for each planting date. To supply deficit fertilizer requirements, $48 \mathrm{~kg} \cdot \mathrm{ha}^{-1}$ of fertilizer were applied for the early and optimal planting date, whilst 51 $\mathrm{kg} \cdot \mathrm{ha}^{-1}$ was applied for the late planting date. Planting rows were opened by hand, $3-5 \mathrm{~cm}$ deep, and seeds were hand-sown in the ground. Thereafter, at crop establishment (14 DAS), seedlings were thinned to the required spacing. Scouting for pests and diseases was done weekly (see ‘Trial layout and design' below). Cypermethrin (15 mL per $10 \mathrm{~L}$ knapsack) was applied to control insect pests 1 month after planting. Weeding was done using hand-hoes at frequent intervals.

\section{Site description}

Field trials were planted at Ukulinga Research Farm $\left(30^{\circ} 24^{\prime}\right.$ S, $29^{\circ} 24^{\prime} \mathrm{E}, 805 \mathrm{~m}$ amsl) on 3 November 2014, 17 November 2014, and 26 January 2015. The farm is situated in Mkhondeni, Pietermaritzburg, in the subtropical hinterland of KwaZulu-Natal Province. Ukulinga represents a semi-arid environment. The soil is classified as Arcadia form, Lonehill family (Soil Classification Working Group, 1991) by South African classification. Rain falls mostly in summer, between September and April. Rainfall distribution varies during the growing season (Swemmer et al., 2007) with the bulk of rain falling in November, December and early January. Occasionally, light to moderate frost occurs in winter (May-July).

\section{Trial layout and design}

The experimental design was a split-plot design with planting date as the main factor and genotypes as the sub-factor, laid out in randomised complete blocks with 3 replicates. The planting dates (3 November 2014, 17 November 2014, and 26 January 2015) represented early, optimal and late planting dates for sorghum. Early planting reflected onset of the rainfall season at Ukulinga. Optimal planting date was based on DAFF (2010) recommendations and historical weather data at Ukulinga. Late planting date represented latest planting for which seasonal rainfall can sustain a 120-140 day growing season, and the prospect of crop maturity before frost or cold stress. Main plot size (a single planting date trial) was $391.5 \mathrm{~m}^{2}$. Sub-plot size (each genotype) was $6 \mathrm{~m} \mathrm{x} 4.5 \mathrm{~m}\left(27 \mathrm{~m}^{2}\right)$, with $1 \mathrm{~m}$ spacing between the plots. Each genotype was replicated 3 times within a single planting date trial. Inter-row spacing was $0.75 \mathrm{~m}$ with $0.30 \mathrm{~m}$ intra-row spacing, amounting to 21 plants per row and 63 experimental plants per plot. Each individual plot had 7 rows with the 3 innermost rows as the experimental plants, and the remaining rows reserved for destructive sampling during growth and development.

\section{Data collection}

\section{Atmospheric data}

Daily data for meteorological parameters were obtained from an on-station (within $100 \mathrm{~m}$ radius) automatic weather station, courtesy of the Agricultural Research Council - Institute for Soil, Climate and Water (ARC-ISCW). Data collected included minimum and maximum temperature, rainfall, maximum and minimum relative humidity, average wind speed and direction, total solar radiation and reference evapotranspiration.

\section{Soil characterisation}

Soil physical and hydraulic properties were obtained from a previous classification and characterisation of experimental site soils by Mabhaudhi (2012). These included volumetric water content at field capacity (FC), permanent wilting point (PWP), and saturation (SAT), as well as saturated hydraulic conductivity $\left(K_{\text {sat }}\right)$, total available water (TAW) and soil depth (Table 1).

\section{Crop data}

Seedling emergence was considered as coleoptile protrusion above soil surface. Weekly emergence was scored from sowing until crop establishment ( $90 \%$ emergence). Final emergence was recorded as maximum seedling emergence obtained from an experimental population. Plant height was measured weekly from establishment, using a tape measure, as distance from soil surface to the tip of the youngest developing leaf (before floral initiation) or tip of the growing panicle (after floral initiation). Leaf number was counted for fully expanded and photosynthetically active (> 50\% green leaf area) leaves from establishment (Mabhaudhi and Modi, 2013). A fully-formed leaf was defined as one where the leaf collar was visible without 


\begin{tabular}{|c|c|c|c|c|c|c|c|c|}
\hline \multicolumn{9}{|c|}{$\begin{array}{c}\text { TABLE } 1 \\
\text { Soil physical and hydraulic properties from Ukulinga experimental site (Mabhaudhi, 2012) }\end{array}$} \\
\hline Soil type & $\begin{array}{c}\text { Clay } \\
\text { content }\end{array}$ & $\begin{array}{l}\text { Soil depth } \\
(\mathrm{m})\end{array}$ & $\begin{array}{l}\text { Bulk density } \\
\left(\mathbf{g} \cdot \mathbf{m}^{-3}\right)\end{array}$ & $\begin{array}{c}\text { Permanent } \\
\text { wilting } \\
\text { point } \\
\left(\mathrm{mm} \cdot \mathrm{m}^{-1}\right)\end{array}$ & $\begin{array}{c}\text { Field } \\
\text { capacity } \\
\left(\mathbf{m m} \cdot \mathbf{m}^{-1}\right)\end{array}$ & $\begin{array}{c}\text { Total } \\
\text { available } \\
\text { water } \\
\left(\mathrm{mm} \cdot \mathrm{m}^{-1}\right)\end{array}$ & $\begin{array}{l}\text { Saturation } \\
\left(\mathbf{m m} \cdot \mathbf{m}^{-1}\right)\end{array}$ & $\begin{array}{c}\text { Saturated } \\
\text { hydraulic } \\
\text { conductivity } \\
\left(\mathrm{mm} \cdot \mathrm{m}^{-1}\right)\end{array}$ \\
\hline Vertisols & \pm 29 & 0.6 & 1.20 & 283.00 & 406.00 & 123.00 & 481.00 & 25.00 \\
\hline
\end{tabular}

dissecting the plant. The flag-leaf was counted as the first leaf upon full formation. Canopy cover (CC) was measured using the LAI2200 canopy analyser (Li-Cor, USA). Values describing the diffuse non-intercepted radiation (DIFN), which is the amount of light visible below the canopy, were taken and converted to percentage canopy cover as described by Mabhaudhi et al. (2014):

$\mathrm{CC}=(1-\mathrm{DIFN}) \times 100 \%$

Chlorophyll content index (CCI) was measured weekly at midday using a SPAD-502 Plus chlorophyll meter (Konica Minolta, Osaka, Japan) on the adaxial surface of the first fully expanded, fully exposed leaf. Stomatal conductance (SC) was measured weekly at midday using a SC-1 leaf porometer (Decagon Devices, USA) from the abaxial surface of the first fully expanded, fully exposed leaf.

\section{Yield and yield-related parameters}

Aboveground dry mass was recorded as the average of two destructively sampled plants per plot. Biomass was recorded as sorghum dry shoot and fruit mass (aboveground marketable biomass) weekly after crop establishment (90\% emergence). Starting at flowering (complete panicle exposure), panicle mass and total above ground above biomass were weighed separately to enable calculations of build-up of harvest index (HI), after which harvest index was calculated as follows:

$H I=Y_{\mathrm{p}} / B$

where: $\quad Y_{\mathrm{p}}=$ panicle mass

$B=$ total aboveground biomass

Total biomass was measured as dry aboveground biomass at physiological maturity. Grain yield and 1000 seed mass were measured at harvest maturity. Thousand seed mass was measured from a random sample of 1000 dry $(<12.5 \%$ moisture content) seeds per genotype. Reference $H I_{0}$ was calculated as follows:

$H I_{\mathrm{o}}=Y_{\mathrm{g}} / B$

where: $\quad Y_{\mathrm{g}}=$ grain yield $\left(\mathrm{kg} \cdot \mathrm{ha}^{-1}\right)$

\section{Phenology}

Time taken to reach a phenological stage was recorded in calendar days and later converted to thermal time (growing degree days, GDD) using Method 2, as described by McMaster and Wilhelm (1997):

$G D D=\Sigma\left[\left(T_{\max }+T_{\min }\right) / 2\right]-T_{\text {base }}$

where: $\quad T_{\max }=$ maximum daily temperature

$T_{\min }=$ minimum daily temperature

$T_{\text {base }}=$ base temperature below which sorghum growth ceases was set at $7^{\circ} \mathrm{C}$

Both Method 1 and Method 2 can be used to calculate GDD. Method 2 individually compares minimum temperature and maximum temperature to base temperature before calculating average temperature. In contrast, Method 1 calculates the average temperature and thereafter compares that to base temperature. Method 2 therefore allows for modification of minimum temperature in relation to base temperature in cases where minimum temperature is below the base temperature. This flexibility is consistent with the requirements of modelling tools such as AquaCrop, thereby simplifying comparisons between actual crop yields and predicted yields.

Time taken to reach a phenological stage was observed as time taken for $50 \%$ of the experimental plant population to exhibit stage diagnostic signals. End of juvenile phase was calculated as the difference between sowing time and flag leaf formation. Floral initiation was marked by a bulging of the plant stem. Flowering was marked by panicle bloom. Full pollen shed by panicles marked onset of anthesis. Duration of flowering was calculated as the time difference from flowering and anthesis. Formation of soft, milky grains after anthesis was observed as the start of grain filling. Appearance of a dark spot on the opposite side of the kernel from the embryo signals completion of dry matter accumulation, hence physiological maturity.

\section{Crop water use}

Soil water content (SWC) was measured every week using a PR2/6 profile probe (Delta-T, UK). Weekly measurements of SWC were then used to compute the soil water balance (Zhao et al., 2004) as follows:

$E T_{a}=I+P+C_{r}-D-R \pm \Delta S W C$

where:

$E T_{\mathrm{a}}=$ crop water use (actual field evapotranspiration)

$I=$ irrigation added $(\mathrm{mm})$

$P=$ rainfall $(\mathrm{mm})$

$C=$ capillary rise $(\mathrm{mm})$

$D=$ drainage $(\mathrm{mm})$

$R=$ run-off

$\triangle S W C=$ change in soil water content 
Since trials were wholly rainfed, there was no irrigation $(I)$ to be considered. Capillary rise $(C)$ and drainage $(D)$ were considered negligible (Ridolfi et al., 2008; Gregory and Northcliff, 2012). Often in calculations of WUE, drainage is considered negligible (Van Halsema and Vincent, 2012) to simplify calculations. In our study, this was also supported by the presence of poorly drained, shallow soils (Table 1). The shallow profile meant that soil water was largely within the effective rooting depth of sorghum supporting the notion of negligible drainage. Runoff $(R)$ was also considered negligible in the soil water balance equation, due to sorghum rows orientated across the slope, limiting runoff to negligible proportions. Therefore, Eq. 5 was simplified to:

$$
E T_{a}=P-\Delta S W C
$$

Actual field evapotranspiration ( $\mathrm{mm}$ ) obtained from Eq. 6 was used to calculate WUE in Eqs. 7 and 8. Water use efficiency refers to the ratio of water used in plant metabolism to water lost by the plant through transpiration and soil evaporation (evapotranspiration). Water use efficiency was calculated for aboveground biomass at physiological maturity, and grain yield at harvest maturity, using the following equations (Kuslu et al., 2010):

Biomass $W U E=B / E T_{a}$

Grain yield $W U E=Y / E T_{a}$

$$
\text { where: } \begin{aligned}
& B=\text { dry aboveground biomass }\left(\mathrm{kg} \cdot \mathrm{ha}^{-1}\right) \\
Y & =\text { grain yield }\left(\mathrm{kg} \cdot \mathrm{ha}^{-1}\right)
\end{aligned}
$$

Growth stages (initial, development and mid-season) were defined according to FAO (1986), and were used to calculate cumulative rainfall received during each stage. Rainfall received for the late season was not considered as crop development was observed from sowing to physiological maturity instead of until harvest maturity.

\section{Data analyses}

Recorded crop parameters were subjected to analyses of variance (ANOVA) using GenStat $16^{\text {th }}$ edition (VSN International, UK) to observe the difference between treatments. Means were separated using least significant differences (LSD) at a probability level of 5\%. Multiple comparisons between means were conducted using Fisher's protected LSDs.

\section{RESULTS AND DISCUSSION}

\section{Crop responses to rainfall, temperature and evapotranspiration}

In South Africa, Du Plessis (2008) reported that, for sorghum, the lower temperature threshold limit was $7^{\circ} \mathrm{C}$, while the upper temperature threshold limit for sorghum grown in the semiarid tropics has been reported as $38^{\circ} \mathrm{C}$ (Huda et al., 1984). Hence $38^{\circ} \mathrm{C}$ and $7^{\circ} \mathrm{C}$ upper and lower temperature thresholds, respectively, were adopted for experimental genotypes in this study. Minimum and maximum temperatures neither exceeded nor went below sorghum growing temperature thresholds during the growing season for the early and optimal planting dates.
This implies that crops did not experience heat or cold stress (Fig. 1). This was confirmed by lack of heat stress symptoms such as scorching of leaves and stems, premature leaf abscission and senescence, shoot growth inhibition or fruit damage (Vollenweider and Günthardt-Goerg, 2005). For late planted sorghum, minimum temperatures dipped below minimum thresholds at 130 DAS (Fig. 1). However, such cold stress coincided with physiological maturity of the sorghum, making the effect on plant growth negligible. Temperatures were relatively low for late planting compared to early and optimal, especially 70 DAS and onwards.
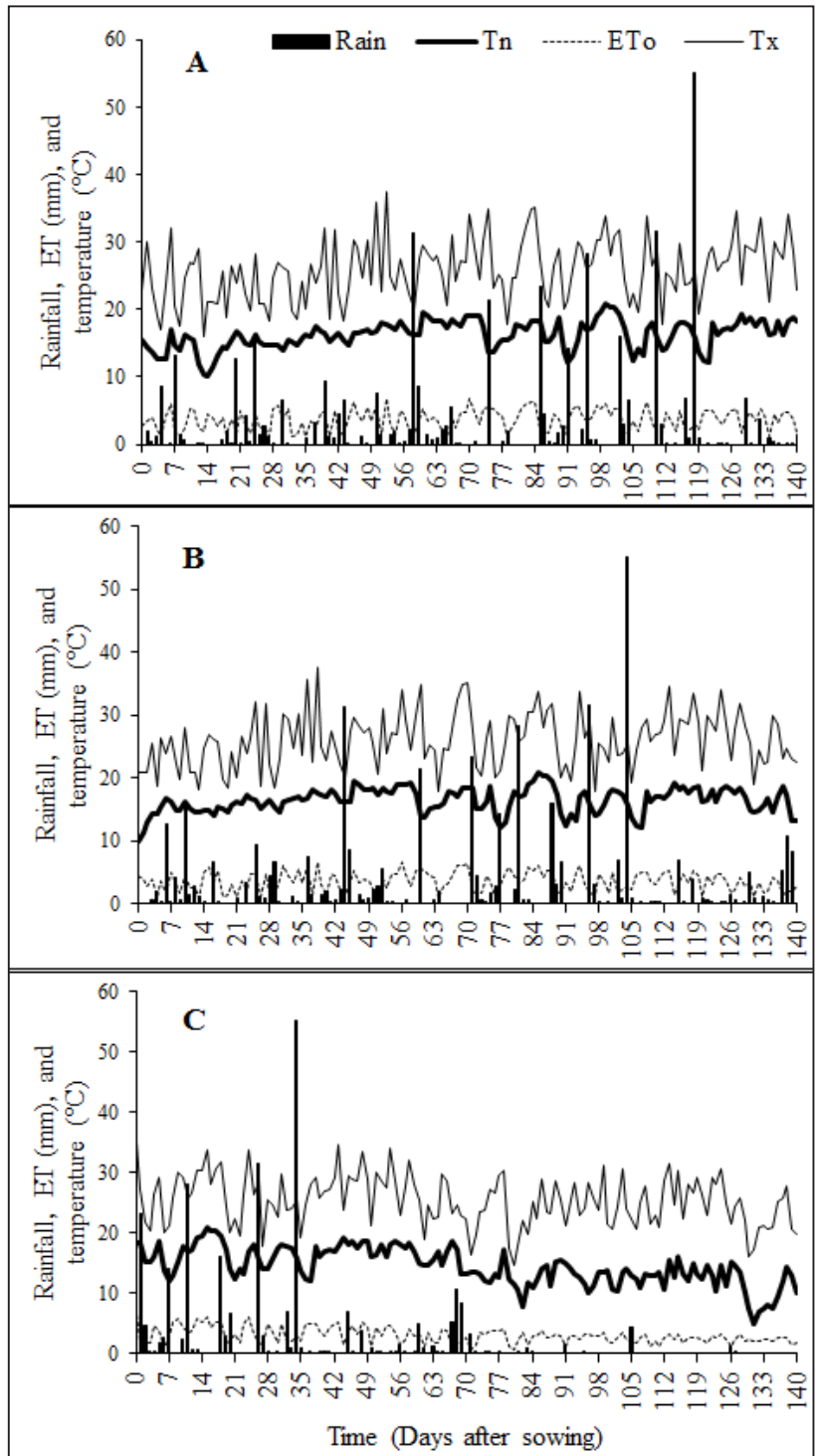

Figure 1

Daily reference evapotranspiration (ETo), minimum temperature (Tn), maximum temperature $(T x)$, and rainfall during the growing seasons of (A) early-, (B) mid-, and (C) late-planted sorghum genotypes

Early planted sorghum received 407.09-418.05 mm, and optimal planting received $389.32-401.25 \mathrm{~mm}$ while late planted sorghum received $266.63-267.13 \mathrm{~mm}$ of rainfall (Table 2). The rainfall ranges depict total rainfall as received by different genotypes due to variations in time to physiological maturity. For all planting dates, recorded rainfall was less than the crop water requirement for sorghum under South African conditions (450-650 mm) (Jewitt et al., 2009). The observed onset of 
rainfall (November) occurred later relative to suggestions by historical data (September). This highlighted climate variability and vulnerability of rainfed agriculture in the region. In this regard, climate forecasts rather than historical data should be recommended to formulate planting dates to synchronize rainfall with growing period. Use of DAFF (2010) guidelines could be misleading as they lack sensitivity to rainfall variability between seasons and assume homogeneity of bio-resource groups (BRGs).

The use of crop models is advantageous in forecasting planting dates across BRGs in SSA; however, availability of soil and climate data for all BRGs remains a challenge to this effect.

Combining the seasonal rainfall prediction with crop water requirement and soil water information is the core component to successful agriculture. Sorghum water requirement ranges from 450-650 mm (Hensley et al., 2000; Jewitt et al., 2009) for optimal yield, assuming a growing season of 120-130 days. Even under sub-optimal seasonal rainfall, optimal crop water use is attained when rainfall distribution between stages is proportionate with crop coefficient $\left(K_{c}\right)$ for water requirements. The synergy between rainfall received per growth stage and $K_{c}$ was least proportionate (Table 3 ) for late planted sorghum, where the least rainfall received was during mid-season stage when crop water requirements are maximum and most rain fell during the initiation stage when crop water requirements are low. On the contrary, early planted sorghum received rainfall proportionate to $K_{c}$ at different growth stages.

\begin{tabular}{|c|c|c|c|c|c|c|c|c|c|}
\hline \multicolumn{10}{|c|}{$\begin{array}{l}\text { TABLE } 2 \\
\text { Water use efficiency (WUE) and water use characteristics at physiological maturity, and seed mass at harvest, of } 4 \text { sorghum } \\
\text { genotypes across } 3 \text { planting dates. }\end{array}$} \\
\hline $\begin{array}{l}\text { Planting } \\
\text { date }\end{array}$ & Genotype & $\begin{array}{l}\text { Rainfall } \\
\text { received } \\
(\mathrm{mm})\end{array}$ & $\begin{array}{l}\text { Water } \\
\text { use } \\
\left(\mathbf{k g} \cdot \mathbf{h a}^{-1}\right)\end{array}$ & $\begin{array}{l}\text { Total yield } \\
\left(\mathbf{k g} \cdot \mathbf{h a}^{-1}\right)\end{array}$ & $\begin{array}{l}\text { Grain } \\
\text { yield } \\
\left(\mathbf{k g} \cdot \mathbf{h a}^{-1}\right)\end{array}$ & $\begin{array}{l}\text { Biomass WUE } \\
\left(\mathbf{k g} \cdot \mathrm{ha}^{-1} \cdot \mathrm{mm}^{-1}\right)\end{array}$ & $\begin{array}{l}\text { Grain Yield WUE } \\
\left(\mathbf{k g} \cdot \mathrm{ha}^{-1} \cdot \mathrm{mm}^{-1}\right)\end{array}$ & $\begin{array}{l}\text { Harvest } \\
\text { index }\end{array}$ & $\begin{array}{c}1000 \\
\text { seed } \\
\text { mass }(g)\end{array}$ \\
\hline \multirow{4}{*}{ 离 } & PAN8816 & 407.09 & 390.5 & $9933.33^{\mathrm{a}}$ & $3813.33^{a}$ & $25.437^{a}$ & $9.765^{\mathrm{a}}$ & $0.383^{\mathrm{a}}$ & $42.47^{\mathrm{g}}$ \\
\hline & Macia & 418.05 & 395.9 & $10613.33^{\mathrm{a}}$ & $4568.89^{\mathrm{ab}}$ & $26.808^{a}$ & $11.541^{\mathrm{ab}}$ & $0.412^{\mathrm{a}}$ & $35.13^{\mathrm{e}}$ \\
\hline & Ujiba & 418.05 & 395.9 & $8884.44^{\mathrm{a}}$ & $2662.22^{\mathrm{bc}}$ & $22.441^{\mathrm{a}}$ & $6.724^{\mathrm{bc}}$ & $0.294^{\mathrm{b}}$ & $31.13^{\mathrm{d}}$ \\
\hline & IsiZulu & 418.05 & 395.9 & $10400.00^{\mathrm{a}}$ & $2004.44^{c}$ & $26.269^{a}$ & $5.063^{c}$ & $0.187^{c}$ & $29.07^{c}$ \\
\hline \multicolumn{2}{|c|}{ Mean } & 415.31 & 394.55 & 9957.78 & 3262.22 & 25.239 & 8.268 & 0.321 & 34.45 \\
\hline \multirow{4}{*}{ 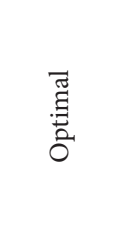 } & PAN8816 & 389.32 & 364.46 & $8946.67^{a}$ & $3426.67^{\mathrm{a}}$ & $24.548^{\mathrm{a}}$ & $9.402^{\mathrm{a}}$ & $0.386^{\mathrm{a}}$ & $38.07^{\mathrm{f}}$ \\
\hline & Macia & 401.25 & 389.56 & $12031.11^{\mathrm{a}}$ & $4782.22^{\mathrm{a}}$ & $30.884^{\mathrm{a}}$ & $12.276^{\mathrm{a}}$ & $0.468^{\mathrm{a}}$ & $35.33^{\mathrm{e}}$ \\
\hline & Ujiba & 389.32 & 364.46 & $9008.89^{\mathrm{ab}}$ & $2591.11^{\mathrm{a}}$ & $24.718^{\mathrm{ab}}$ & $7.109^{\mathrm{a}}$ & $0.286^{\mathrm{b}}$ & $28.20^{\mathrm{bc}}$ \\
\hline & IsiZulu & 393.12 & 364.66 & $15262.22^{b}$ & $2768.89^{b}$ & $41.853^{\mathrm{b}}$ & $7.593^{\mathrm{b}}$ & $0.182^{c}$ & $28.13^{\mathrm{bc}}$ \\
\hline \multicolumn{2}{|c|}{ Mean } & 393.25 & 370.79 & 11312.22 & 3392.22 & 30.501 & 9.149 & 0.330 & 34.43 \\
\hline \multirow{4}{*}{ 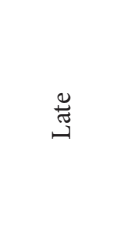 } & PAN8816 & 266.63 & 237.61 & $4533.33^{a}$ & $1804.44^{\mathrm{a}}$ & $19.079^{\mathrm{a}}$ & $7.594^{\mathrm{a}}$ & $0.402^{\mathrm{a}}$ & $31.27^{\mathrm{d}}$ \\
\hline & Macia & 266.88 & 237.86 & $5746.67^{\mathrm{a}}$ & $2191.11^{\mathrm{a}}$ & $24.185^{\mathrm{a}}$ & $9.212^{\mathrm{a}}$ & $0.382^{\mathrm{a}}$ & $26.83^{\mathrm{ab}}$ \\
\hline & Ujiba & 266.63 & 237.61 & $6293.33^{\mathrm{a}}$ & $2306.67^{\mathrm{a}}$ & $26.486^{\mathrm{a}}$ & $9.708^{\mathrm{a}}$ & $0.371^{\mathrm{a}}$ & $25.40^{\mathrm{a}}$ \\
\hline & IsiZulu & 266.63 & 237.61 & $5417.78^{a}$ & $1991.11^{\mathrm{a}}$ & $22.801^{\mathrm{a}}$ & $8.380^{\mathrm{a}}$ & $0.373^{\mathrm{a}}$ & $26.17^{a}$ \\
\hline \multicolumn{2}{|c|}{ Mean } & 266.69 & 237.61 & 5497.78 & 2073.33 & 23.138 & 8.726 & 0.382 & 27.42 \\
\hline \multicolumn{4}{|c|}{$\mathrm{LSD}_{(\mathrm{P}=0.05)}[$ Planting date $\mathrm{x}$ Genotype $]$} & 185.29 & 679.1 & 2.834 & 2.128 & 0.052 & 1.92 \\
\hline \multicolumn{4}{|c|}{$\% \mathrm{CV}$ [Planting date $\mathrm{x}$ Genotype] } & 12.5 & 13.8 & 8.7 & 14.4 & 8.9 & 3.6 \\
\hline
\end{tabular}

${ }^{a}$ Values within a column sharing the same alphabet letters represent statistically the same yields and water use characteristics, whilst different alphabet letters represent significant different in yields and water use characteristics across interaction of planting dates and genotypes.

\begin{tabular}{|c|c|c|c|c|}
\hline \multicolumn{5}{|c|}{$\begin{array}{l}\text { TABLE } 3 \\
\text { Rainfall received at } 3 \text { key developmental stages when planted on } 3 \text { planting dates. }\end{array}$} \\
\hline \multirow{2}{*}{ Planting dates } & \multicolumn{3}{|c|}{ Rainfall (mm) received during each growth stage } & \multirow[t]{2}{*}{ Total } \\
\hline & Initial & Development & Mid-season & \\
\hline Early & 79.47 & 151.11 & 187.43 & 418.05 \\
\hline Optimal & 78.71 & 173.20 & 174.46 & 401.25 \\
\hline Late & 204.19 & 40.86 & 22.08 & 267.13 \\
\hline ¥Crop co-efficient $(K c)$ & 0.45 & 0.83 & 1.18 & \\
\hline
\end{tabular}

¥Obtained from Shenkut et al. (2013). 


\begin{tabular}{|c|c|c|c|c|c|c|c|c|}
\hline \multicolumn{9}{|c|}{$\begin{array}{c}\text { TABLE } 4 \\
\text { Time (in calendar and growing degree days) taken for } 4 \text { sorghum genotypes planted at } 3 \text { different planting dates to reach } \\
\text { particular phenological stages. }\end{array}$} \\
\hline \multirow{2}{*}{ Stage } & \multirow{2}{*}{$\begin{array}{l}\text { Planting } \\
\text { date }\end{array}$} & \multicolumn{5}{|c|}{ Time taken to reach phenological stage in days (growing degree days) } & \multirow{2}{*}{$\operatorname{LSD}_{(P=0.05)}$} & \multirow{2}{*}{$\% \mathrm{CV}$} \\
\hline & & Ujiba & Macia & PAN8816 & IsiZulu & Mean & & \\
\hline \multirow{3}{*}{$\mathrm{CE}$} & Early & $21(257.1)^{\mathrm{c}}$ & $21(257.1)^{\mathrm{c}}$ & $14(173.6)^{\mathrm{b}}$ & $14(173.6)^{\mathrm{b}}$ & 17.5 & \multirow{3}{*}{1.98} & \multirow{3}{*}{2.1} \\
\hline & Optimal & $28(357.1)^{\mathrm{d}}$ & $21(257.9)^{c}$ & $14(176.1)^{a}$ & $28(357.1)^{\mathrm{d}}$ & 22.2 & & \\
\hline & Late & $7(107)^{\mathrm{a}}$ & $14(215.9)^{\mathrm{b}}$ & $7(107)^{\mathrm{a}}$ & $7(107)^{\mathrm{a}}$ & 8.8 & & \\
\hline \multirow{3}{*}{ EJ } & Early & $91(1246.0)^{\mathrm{e}}$ & $91(1246.0)^{\mathrm{e}}$ & $77(1048.1)^{c}$ & $91(1246.0)^{\mathrm{e}}$ & 87.5 & \multirow{3}{*}{1.98} & \multirow{3}{*}{0.4} \\
\hline & Optimal & $84(1190.1)^{d}$ & $91(1298.1)^{\mathrm{e}}$ & $77(1081.0)^{c}$ & $91(1298.1)^{\mathrm{e}}$ & 82.3 & & \\
\hline & Late & $63(933.7)^{a}$ & $70(1025.3)^{b}$ & $63(933.7)^{\mathrm{a}}$ & $63(933.7)^{\mathrm{a}}$ & 67.7 & & \\
\hline \multirow{3}{*}{ FI } & Early & $98(1355.2)^{\mathrm{b}}$ & $98(1355.2)^{\mathrm{b}}$ & $84(1 \quad 158.9)^{\mathrm{a}}$ & $98(1355.2)^{\mathrm{b}}$ & 94.5 & \multirow{3}{*}{2.03} & \multirow{3}{*}{0.6} \\
\hline & Optimal & $91(1298.1)^{b}$ & $98(1390.3)^{c}$ & $84(1 \quad 190.1)^{a}$ & $98(1390.3)^{c}$ & 92.8 & & \\
\hline & Late & $70\left(\begin{array}{lll}1 & 025.3)^{\mathrm{a}}\end{array}\right.$ & $77(1 \quad 104.4)^{\mathrm{b}}$ & $70(1025.3)^{\mathrm{a}}$ & $70(1025.3)^{\mathrm{a}}$ & 75.3 & & \\
\hline \multirow{3}{*}{$\mathrm{F}$} & Early & $105(1463.1)^{\mathrm{e}}$ & $105(1463.1)^{\mathrm{e}}$ & $91(1246.3)^{e}$ & $105\left(\begin{array}{l}1463.1) \\
\mathrm{e}\end{array}\right.$ & 101.5 & \multirow{3}{*}{1.98} & \multirow{3}{*}{0.3} \\
\hline & Optimal & $98(1390.3)^{\mathrm{d}}$ & $105(1483.2)^{c}$ & $91(1298.1)^{c}$ & $105\left(\begin{array}{l}483.2) \\
c\end{array}\right.$ & 99.8 & & \\
\hline & Late & $84(1170.3)^{b}$ & $84(1 \quad 170.3)^{b}$ & $77(1 \quad 104.4)^{\mathrm{a}}$ & $84(1170.3)^{\mathrm{b}}$ & 82.3 & & \\
\hline \multirow{3}{*}{ FD } & Early & $7(92.4)^{\mathrm{a}}$ & $7(92.4)^{\mathrm{a}}$ & $7(108.9)^{a}$ & $7(92.4)^{a}$ & 7 & \multirow{3}{*}{-} & \multirow{3}{*}{-} \\
\hline & Optimal & $7(92.9)^{\mathrm{a}}$ & $7(101.2)^{a}$ & $7(92.2)^{\mathrm{a}}$ & $7(101.2)^{\mathrm{a}}$ & 7 & & \\
\hline & Late & $7(85.3)^{\mathrm{a}}$ & $7(85.3)^{\mathrm{a}}$ & $7(65.9)^{\mathrm{a}}$ & $7(85.3)^{\mathrm{a}}$ & 7 & & \\
\hline \multirow{3}{*}{ A } & Early & $112(1555.4)^{\mathrm{b}}$ & $112(1555.4)^{\mathrm{b}}$ & $98(1355.2)^{\mathrm{b}}$ & $112\left(\begin{array}{r}1555.5) \\
\mathrm{b}\end{array}\right.$ & 108.5 & \multirow{3}{*}{2.00} & \multirow{3}{*}{0.6} \\
\hline & Optimal & $105(1483.2)^{\mathrm{b}}$ & $112(1584.4)^{\mathrm{b}}$ & $98(1390.3)^{\mathrm{b}}$ & $112\left(\begin{array}{r}584.4) \\
b\end{array}\right.$ & 106.8 & & \\
\hline & Late & $91(1256.2)^{b}$ & $91(1256.2)^{\mathrm{b}}$ & $84(1 \quad 170.3)^{\mathrm{a}}$ & $91(1256.2)^{\mathrm{b}}$ & 89.3 & & \\
\hline \multirow{3}{*}{ GF } & Early & $119(1648.2)^{\mathrm{b}}$ & $119(1648.2)^{\mathrm{b}}$ & $105\left(\begin{array}{l}1 \\
1463.1)\end{array}\right.$ & $119\left(\begin{array}{l}1 \\
648.2)\end{array}\right.$ & 115.5 & \multirow{3}{*}{1.98} & \multirow{3}{*}{0.6} \\
\hline & Optimal & $112(1584.4)^{\mathrm{b}}$ & $119(1703.6)^{c}$ & $105\left(\begin{array}{r}483.2) \\
\mathrm{a}\end{array}\right.$ & $119\left(\begin{array}{r}703.6) \\
c\end{array}\right.$ & 113.8 & & \\
\hline & Late & $98(1351.2)^{\mathrm{b}}$ & $98(1351.2)^{\mathrm{b}}$ & $91(1256.2)^{\mathrm{a}}$ & $98(1351.2)^{\mathrm{b}}$ & 96.3 & & \\
\hline \multirow{3}{*}{ PM } & Early & $140(1976.3)^{\mathrm{b}}$ & $140(1976.3)^{b}$ & $133\left(\begin{array}{l}1 \\
1868.6)\end{array}\right.$ & $140(1976.3)$ & 138.3 & \multirow{3}{*}{1.76} & \multirow{3}{*}{0.4} \\
\hline & Optimal & $126(1811.2)^{\mathrm{a}}$ & $140(1999.5)^{\mathrm{c}}$ & $126\left(\begin{array}{l}1 \\
1\end{array}\right.$ & $133\left(\begin{array}{r}1907.9) \\
b\end{array}\right.$ & 131.3 & & \\
\hline & Late & $126(1697.9)^{a}$ & $133(1756.5)^{c}$ & $126\left(\begin{array}{r}1 \\
6997.9) \\
a\end{array}\right.$ & $126\left(\begin{array}{r}1 \\
697.9) \\
b\end{array}\right.$ & 127.8 & & \\
\hline
\end{tabular}

CE (crop establishment); EJ (end of juvenile); FI (floral initiation); F (flowering); FD (flowering duration); A (anthesis); GF (start of grain filling); PM (physiological maturity).

a Values sharing the same letter represent statistically similar yields and water use characteristics, whilst different letters represent significantly different yields and water use characteristics at each planting date. 


\section{Final emergence and crop establishment}

The interaction between planting date and genotype was highly significant $(P<0.001)$ with respect to final emergence (Table 4; Fig. 3). Full emergence (100\%) was achieved for all genotypes when sorghum was planted late. Consequently, crop establishment was fastest for the late planting date. Early establishment and high seedling emergence for all genotypes for late planted sorghum were attributed to high cumulative rainfall 7 days after sowing and high initial soil water content at sowing. At least $25 \mathrm{~mm}$ of rainfall should fall within a 7-day period after sowing (Raes et al., 2004; Mhizha et al., 2014) for early and optimal emergence. Final emergence was significantly lower for Macia (57.6\%), whilst other genotypes attained full establishment in response to early planting. Low initial SWC at sowing (Fig. 2) resulted in delayed crop establishment for early planted sorghum for all genotypes compared to optimal and late planting dates. For all three planting dates, PAN8816 achieved full emergence, although there were variations in times to emergence. Time to crop establishment was shown to be significantly $(P<0.001)$ slower and lower for the optimal planting date (Table 4); this trend was clearer for Macia (31.2\% emergence 21 DAS) relative to other sorghum genotypes (Fig. 3).

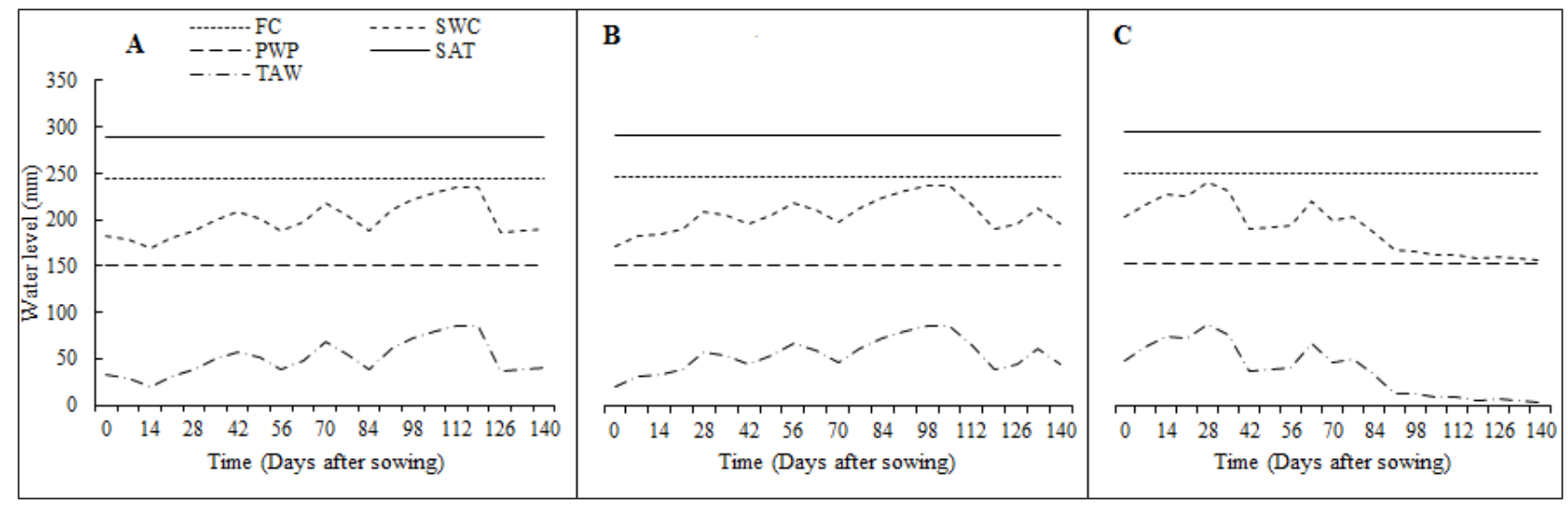

Figure 2

Soil hydraulic properties over time during the growing seasons of early $(A)$, optimal $(B)$, and late $(C)$ planted sorghum. Note: $F C=$ field capacity; $P W P=$ permanent wilting point; SWC = soil water content; TAW = total available water; SAT = saturation point .
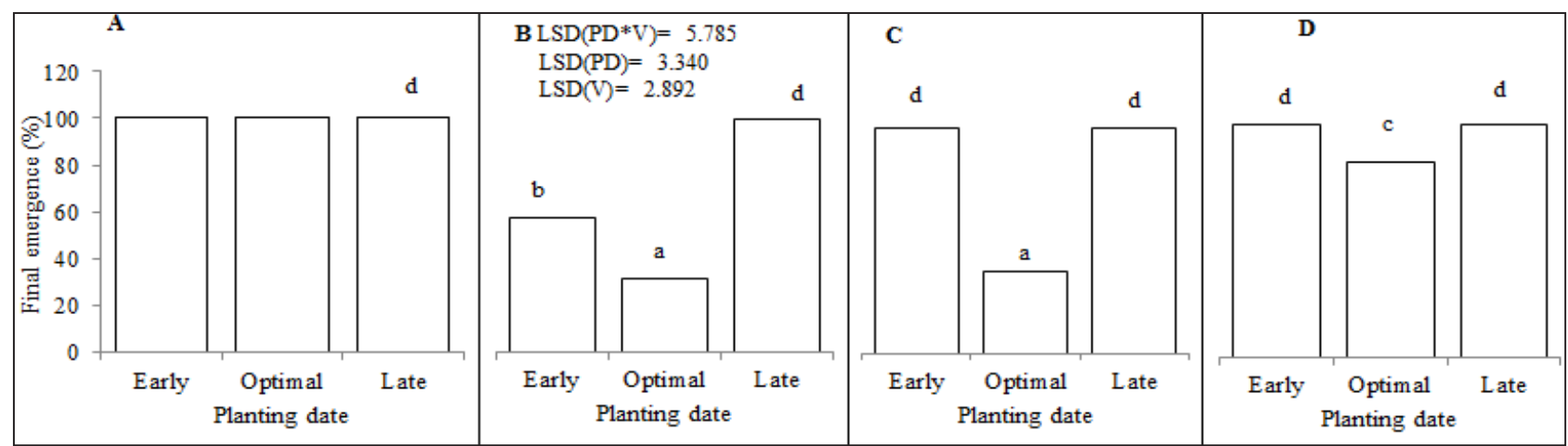

Figure 3

Final percentage emergence of sorghum genotypes (A) PAN8816, (B) Macia, (C) Ujiba, and (D) IsiZulu] planted at Ukulinga on 3 planting dates. Note: $P D^{*} V$ refers to the interaction between planting dates $(P D)$ and sorghum genotypes $(V)$, respectively. Values sharing the same letter are similar at $L S D$ $\left(P D^{*} V\right)=0.05$.

\section{CROP MORPHOLOGY AND PHYSIOLOGY}

Late-planted sorghum genotypes exhibited lower $(P<0.001)$ average leaf number, CCI and SC $(5.8 ; 47.22 ; 273.3 \mathrm{mmol} \cdot \mathrm{m}$ $\left.{ }^{2} \cdot \mathrm{s}^{-1}\right)$ compared to early $\left(7.7 ; 50.57 ; 298.7 \mathrm{mmol} \cdot \mathrm{m}^{-2} \cdot \mathrm{s}^{-1}\right)$ and optimal $\left(7.3 ; 51.74 ; 323.0 \mathrm{mmol} \cdot \mathrm{m}^{-2} \cdot \mathrm{s}^{-1}\right)$ planting, respectively. This was due to low SWC and TAW observed during the late planting date (Saddam et al., 2014). This was more pronounced post floral initiation due to low and irregular rainfall during the mid-season stage. Leaf number and CCI were statistically similar between early and optimal planting dates (Fig. 4). This was attributed to similarities in climate due to closeness between the two planting dates, which highlights the need to use area-specific climate forecasting instead of historical data and countrywide recommendations for planting date selections. Sorghum genotypes maintained statistically similar $(P>0.05)$ CCI (Fig. 7) and stomatal conductance (Fig. 8) across planting dates. However, leaf number varied significantly among genotypes $(P<0.001$; Fig. 4$)$. Highest leaf number was observed for PAN8816 (7.4) followed by Macia (7.2). Leaf numbers were lowest and statistically similar for IsiZulu (6.6) and Ujiba (6.5) landraces. Leaf number variations across genotypes were attributed to genotypic differences. Chlorophyll content index increased and plateaued after floral initiation with peak CCI measurements ranging between 50 and 61; these results were similar to observations by Van Oosterom et al. (2010). Stomatal conductance in landraces was less sensitive to differences in TAW compared to the OPV and hybrid genotypes. Results of 
leaf number, CCI and SC suggested that sorghum was subjected to significant water stress, especially when planted late. This was consistent with observations of soil water content which showed a decreasing trend from 84 DAS (Fig. 2), and irregular and erratic rainfall after 70 DAS (Fig. 1) which showed that soil water availability and rainfall increasingly became limiting for the late planting date.

Growth indicators of plant height and leaf number increased gradually until flowering and end of vegetative stage, respectively. Increase in plant height in sorghum occurred mostly during the vegetative stage, and full panicle formation coincided with attainment of maximum plant height (Fig. 5). Planting dates and varietal differences significantly $(P<0.05)$ affected plant height. Late planted sorghum grew tallest for all genotypes, albeit with fewer leaves relative to the other planting dates. An exception was the IsiZulu landrace, where plant height reduced at late planting, resulting in decreased biomass and markedly improved HI. Longer but thinner shoots were associated with a lower biomass accumulation under severe water stress for the late planting date. This suggests that plant height, stem diameter and leaf number form key adaptations of shoot growth to water stress in sorghum.

Canopy cover largely relies on crop density and amount of plant leaf material. Increases in CC limit water loss from soil by evaporation, whilst improving the water portion allocated to crop biomass production via transpiration (Mabhaudhi et al., 2013). Variations in measured CC were significant for both planting dates $(P<0.001)$ and genotypes $(P<0.05)$. Canopy cover for the early planted crop benefitted from good crop stand and high leaf number. Low CC for the late planted crop was associated with low leaf number. Canopy cover was lowest at optimal planting date due to low crop stand. Decreased crop stand in landrace and OPV genotypes and delayed emergence contributed mostly to observed low CC (Fig. 7). It may also be that the low canopy cover at the late planting date allowed for more soil evaporation to occur. This may have inadvertently worsened the situation of low SWC and TAW as less soil water was available for productive water loss (transpiration).

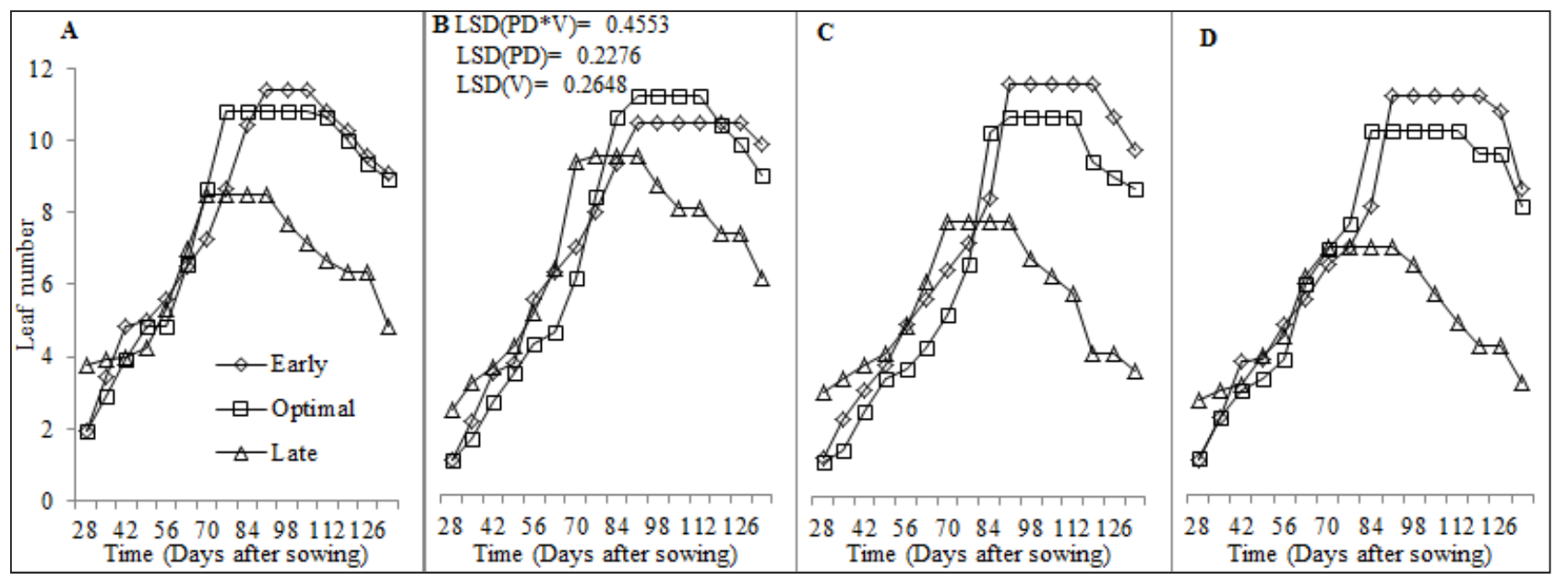

\section{Figure 4}

Leaf number progressions observed after establishment in PAN8816 (A), Macia (B), Ujiba (C) and IsiZulu (D) sorghum genotypes planted on different planting dates. Note: $P D^{*} V$ refers to the interaction between planting dates $(P D)$ and sorghum genotypes $(V)$, respectively.

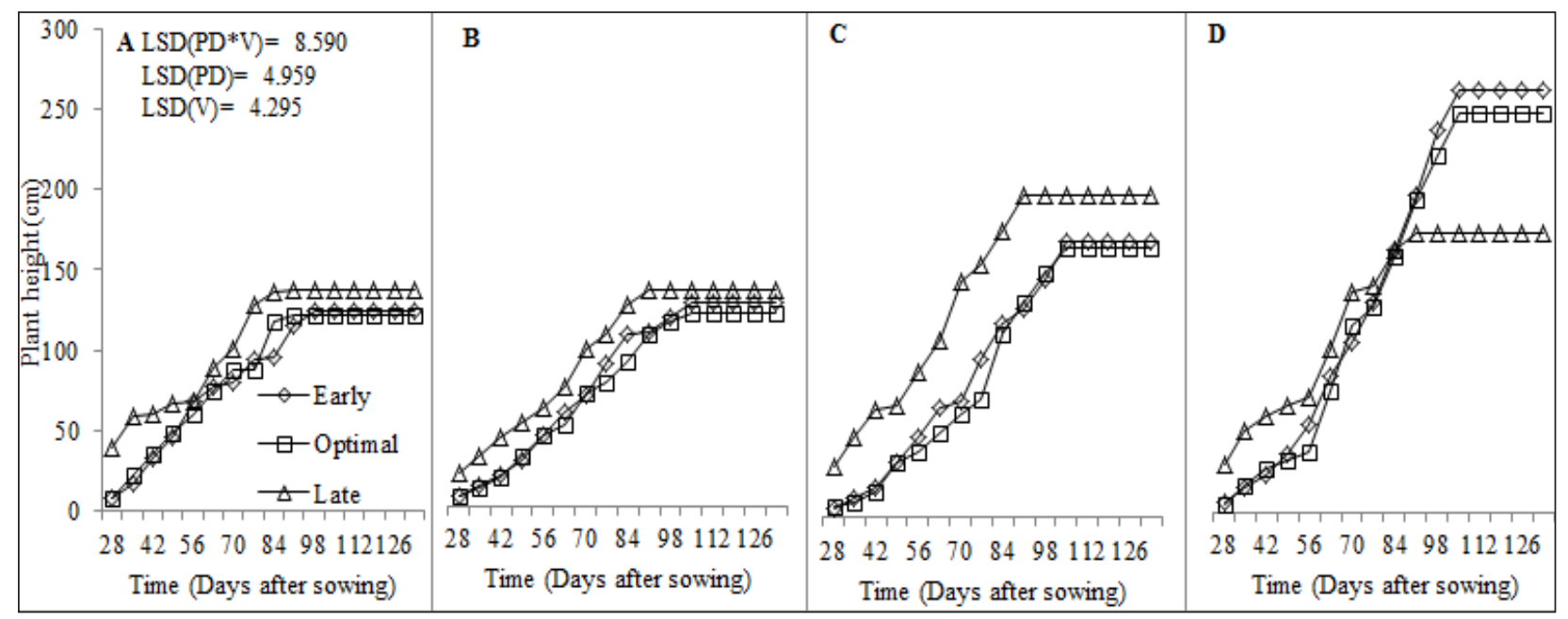

Figure 5

Plant height observed after establishment in PAN8816 (A), Macia (B), Ujiba (C) and IsiZulu (D) sorghum genotypes planted on different planting dates. Note: $P D^{*} V$ refers to the interaction between planting dates $(P D)$ and sorghum genotypes $(V)$, respectively. 


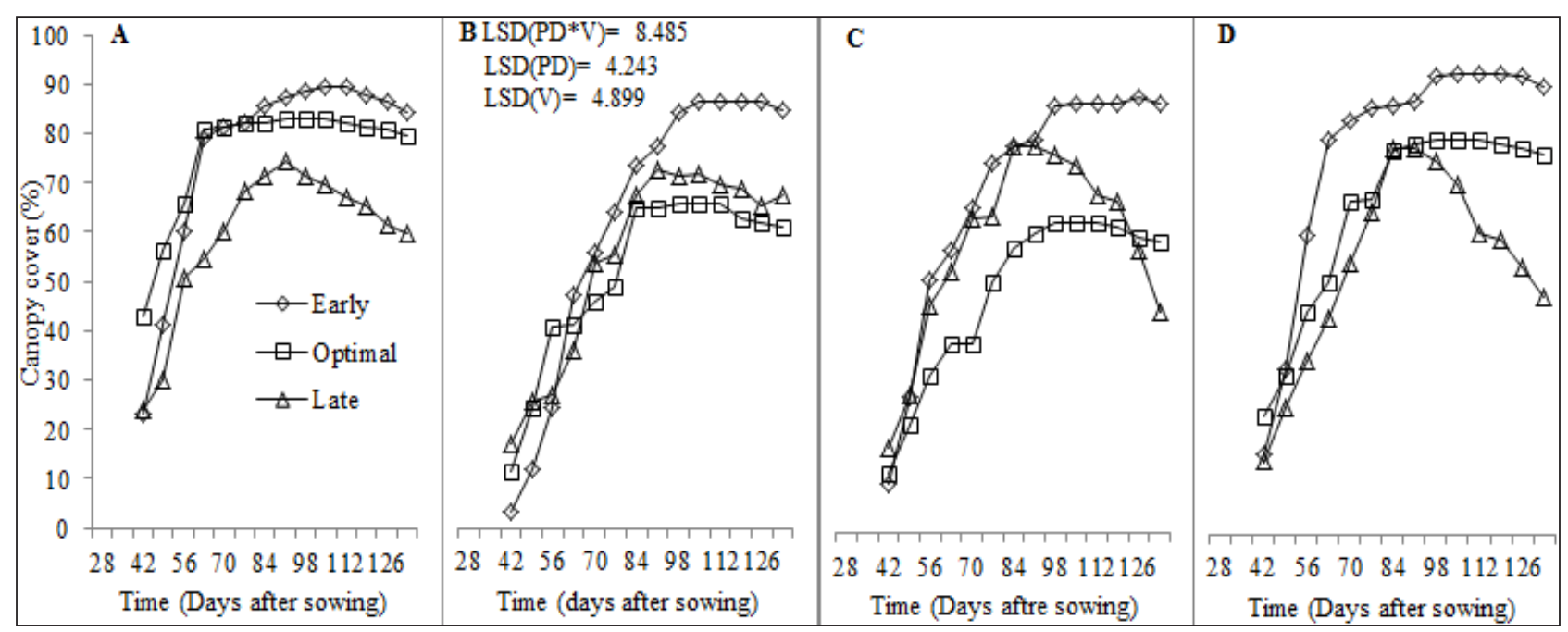

Figure 6

Percentage canopy cover in PAN8816 (A), Macia (B), Ujiba (C) and IsiZulu (D) sorghum genotypes planted on different planting dates. Note: PD*V refers to the interaction between planting dates $(P D)$ and sorghum genotypes $(V)$, respectively.

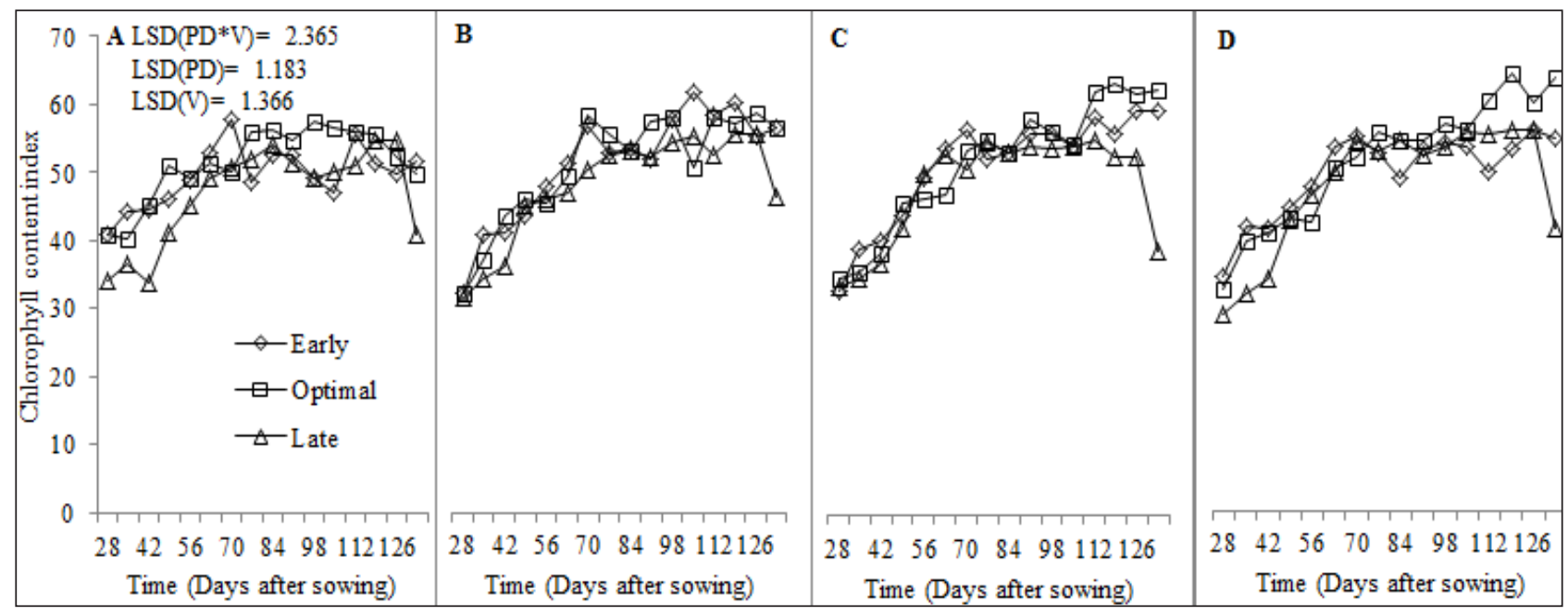

Figure 7

Chlorophyll content index in PAN8816 (A), Macia (B), Ujiba (C) and IsiZulu (D) sorghum genotypes planted on different planting dates from establishment until maturity. Note: $P D^{*} V$ refers to the interaction between planting dates $(P D)$ and sorghum genotypes $(V)$, respectively.

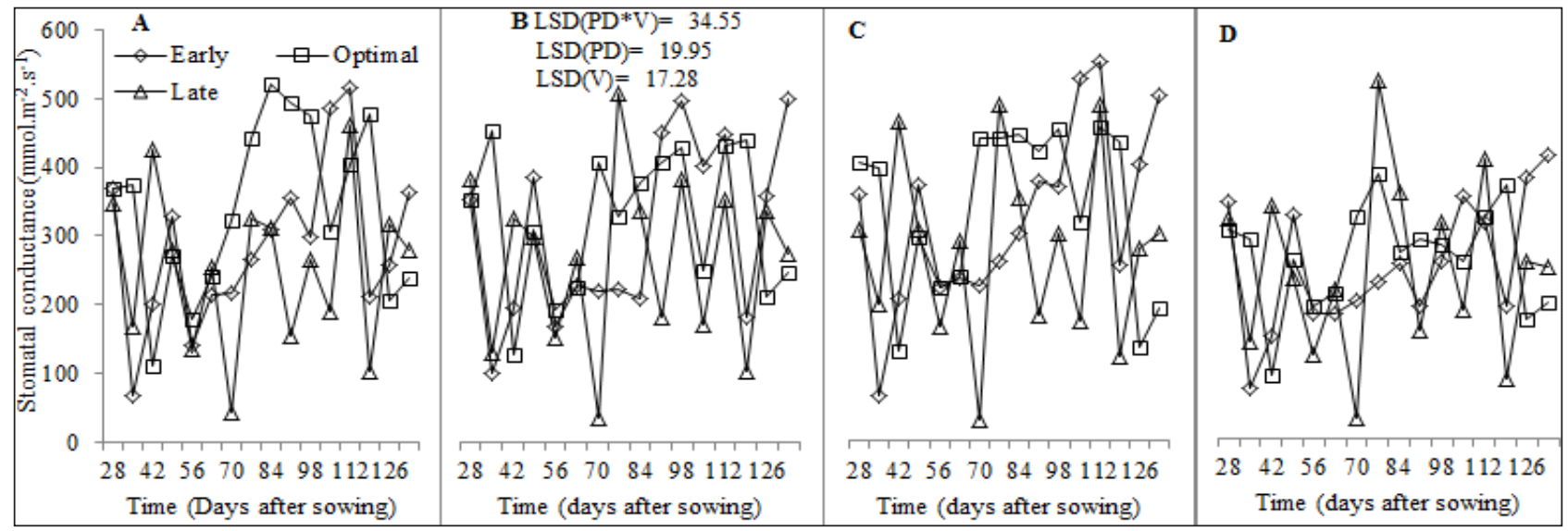

Figure 8

Stomatal conductance in PAN8816 (A), Macia (B), Ujiba (C) and IsiZulu (D) sorghum genotypes planted on different planting dates from establishment until maturity. Note: $P D^{*} V$ refers to the interaction between planting dates $(P D)$ and sorghum genotypes $(V)$, respectively. 


\section{Phenological development and yield}

Early planting generally resulted in delayed phenological development and maturity, resulting in a longer (Table 4) but slower grain filling duration as depicted by harvest index accumulation. Hybrid and landrace genotypes had a significantly $(P<0.05)$ shorter growing season relative to the OPV for all planting dates. It could be argued that Macia employs quiescence strategies to a greater degree relative to the hybrid and landrace genotypes to adapt to irregular rainfall and water scarcity, hence delays in time to physiological maturity. Genotypes that employ quiescence are expected to delay rather than hasten physiological maturity under severe water stress, and low, irregular rainfall. However, observed shortening of growing cycle for late planting date under severe water stress for the late planting date suggests that Macia is a late maturing genotype. This explains the consistently longer growing cycle in Macia compared to the hybrid and landrace genotypes. Phenological development hastened for all genotypes under late planting in order to escape both pre- and post-anthesis water stress. This resulted in early flowering, decreased grain and biomass yield at late planting. Water stress potentially resulted in decreased head size at floral initiation, flower abortion at flowering, and less assimilates for grain filling. This resulted in reduced seed mass and grain yield in late planted sorghum (Table 2). Early and optimal planting dates resulted in significantly $(P<0.001)$ higher 1000 -seed mass (34.5 and $34.4 \mathrm{~g}$ ), compared to late planting (27.4 g). Thousand seed mass was linked to water availability in the mid-season stage (Table 3), which directly affected photosynthesis and yield accumulation at grain filling stage.

Tall growing genotypes consistently had higher dry biomass accumulation (Fig. 9) and, in comparison, small source to sink biomass partitioning (Fig. 10), resulting in lower harvest index (Fig. 11). In general, build-up of harvest index (Fig. 11) and reference harvest index (Table 2) improved with decreasing rainfall, reinforcing sorghum as an exemplar drought-tolerant cereal for production in arid and semi-arid regions. The improvement in $\mathrm{HI}$ under stress highlights that water stress has a positive effect on HI of sorghum. This implies that, when subjected to stress, the crop will partition more of its assimilates to the yield component, which is a desirable characteristic under rainfed conditions. The isiZulu landrace decreased shoot (plant height) growth to increase source to sink biomass partitioning under severe water stress at late planting. This resulted in twofold

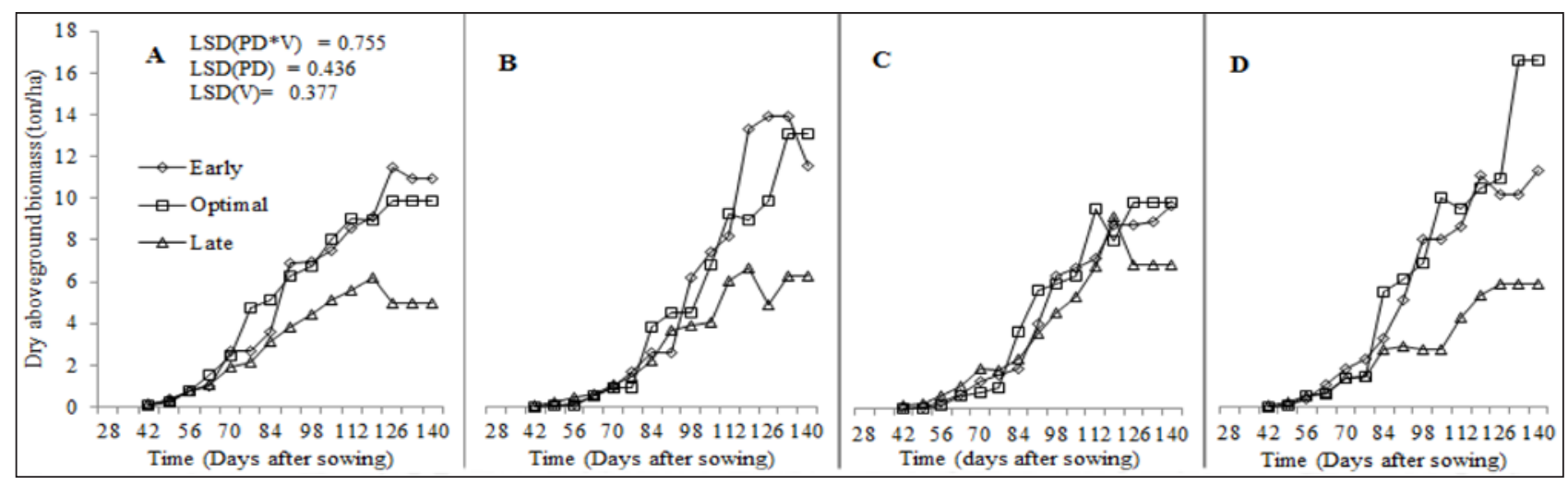

Figure 9

Destructively sampled dry aboveground biomass of PAN8816 (A), Macia (B), Ujiba (C) and IsiZulu (D) sorghum genotypes planted on 3 different planting dates. Note: $P D^{*} V$ refers to the interaction between planting dates $(P D)$ and sorghum genotypes $(V)$, respectively.

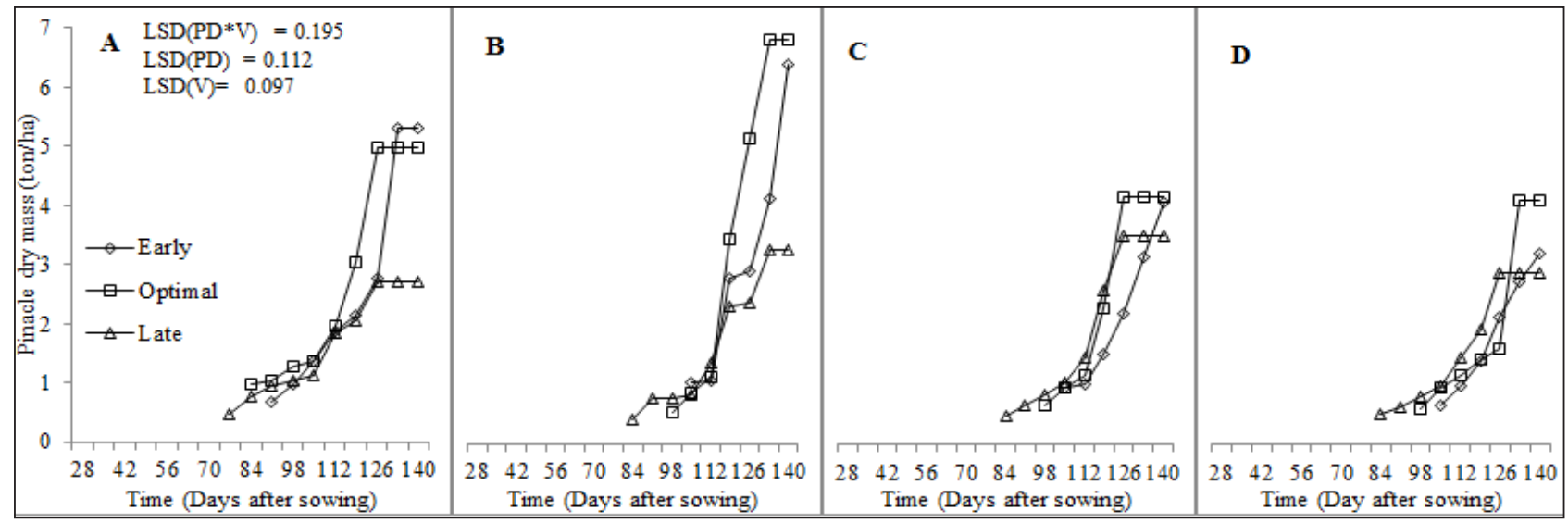

Figure 10

Destructively sampled pinnacle mass of PAN8816 (A), Macia (B), Ujiba (C) and IsiZulu (D) sorghum genotypes planted on 3 different planting dates. Note: $P D^{*} V$ refers to the interaction between planting dates $(P D)$ and sorghum genotypes $(V)$, respectively. 
increases in $H I_{0}$, highlighting high adaptation of the landrace to low and irregular rainfall. At early and optimal planting, highest grain yield (Fig. 10) and harvest index (Fig. 11) were observed in Macia, due to extended grain filling period and late maturity. This shows adaptation of Macia OPV to suboptimal rainfall regions and the benefit of deliberate breeding for drought tolerance in sorghum. However, late planting significantly reduced Macia yield, implying that further breeding for severe water stress and periodic rainfall need to be conducted for Macia. Low sorghum biomass and grain yields at late planting were attributed to low leaf number, CCI, SC and CC in response to low and disproportionate rainfall. This was exacerbated by the fact that flowering coincided with declining rainfall and temperatures as winter set in.

Landraces used in this study showed grain yield stability across planting dates, whilst Macia and PAN8816 genotypes had significantly less grain yield in response to decreased water availability and erratic rainfall distribution at late planting. This suggests that landraces have greater environmental plasticity compared to the OPV and hybrid genotypes in this study. Ujiba and IsiZulu landraces therefore have yield advantages under severe water stress and uneven rainfall distribution; however, at relatively low water stress, the PAN8816 hybrid and Macia OPV confer a yield advantage. Continued landrace cultivation in semi-arid, rainfed conditions by small-scale farmers makes landraces particularly suited for production in low-rainfall areas, and a valuable germplasm resource in breeding for drought tolerance.

\section{Water use and WUE}

Water use was sub-optimal for all planting dates; this was associated with below-average rainfall received. Measured crop water use was exceptionally low, confirming severe water stress experienced by genotypes for the late planting date. The interaction effect of planting dates and genotypes was significant $(P<0.001)$ for biomass and grain yield WUE. Biomass and grain yield WUE were highest at optimal planting (30.5 and $\left.9.2 \mathrm{~kg} \cdot \mathrm{ha}^{-1} \cdot \mathrm{mm}^{-1}\right)$, relative to late $\left(23.1\right.$ and $\left.8.7 \mathrm{~kg} \cdot \mathrm{ha}^{-1} \cdot \mathrm{mm}^{-1}\right)$, and early planting dates $\left(25.2\right.$ and $\left.8.3 \mathrm{~kg} \cdot \mathrm{ha}^{-1} \cdot \mathrm{mm}^{-1}\right)$. For the PAN8816 and Macia, biomass and yield WUE decreased in response to low TAW, and irregular and disproportionate rainfall experienced during the late planting date. By contrast, biomass and grain yield WUE for the IsiZulu and Ujiba landraces improved with decreasing rainfall (Table 2). This reinforces findings in this study that landraces are highly suitable for production under severe water stress, as more 'food per drop' is produced with less rainfall. Grain yield WUE for all genotypes and planting dates were sub-optimal in comparison to optimal values (12.4-13.4 kg.ha-1 $\mathrm{mm}^{-1}$ ) reported by Maman et al. (2013). This implies that a higher decrease in grain yield occurred in relation to lowered water availability for all planting dates and genotypes. A well-watered treatment would have provided a fair comparison of optimal WUE at Ukulinga given that genotypes used, geographical, climatic and soil conditions were different to those reported by Maman et al. (2013).

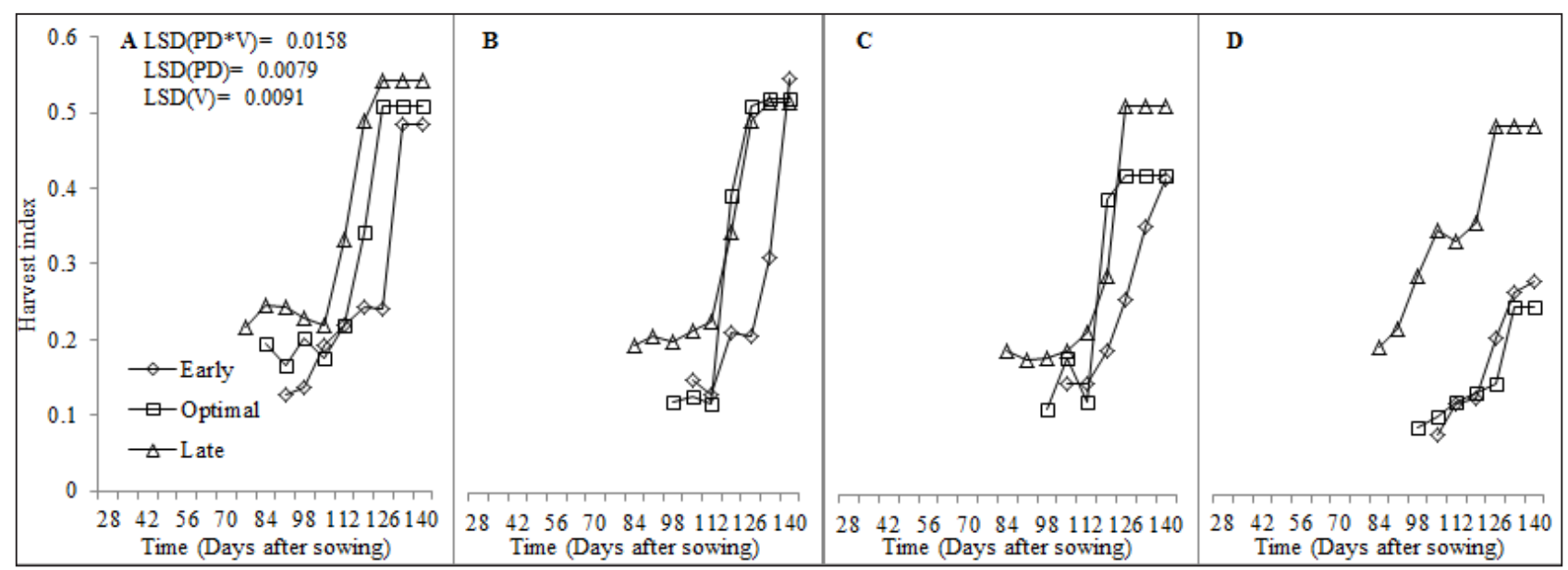

Figure 11

Build-up of harvest indices in PAN8816 (A), Macia (B), Ujiba (C) and IsiZulu (D) sorghum genotypes planted on 3 different planting dates. Note: PD*V refers to the interaction between planting dates $(P D)$ and sorghum genotypes $(V)$, respectively.

\section{CONCLUSIONS}

Rainfall received for all planting dates was suboptimal. Rainfall for the late planting was relatively low, highly irregular and disproportionate to crop water requirements at key growth stages. Low TAW at planting was associated with delayed crop establishment and low final emergence. The exception was PAN8816 hybrid which achieved full emergence for all planting dates. All genotypes adapted to low and irregular rainfall by decreasing CC, CCI and SC, and hastened phenological development. This resulted in low biomass and yield for late planted sorghum. The landraces Ujiba and IsiZulu showed grain yield stability across planting dates, whilst the OPV Macia and hybrid PAN8816 varieties had significantly lower grain yield under low soil water availability and uneven rainfall distribution experienced for the late planting date. Biomass and grain yield WUE were highest for the optimal planting date relative to the late, and lowest at early planting dates. For PAN8816 and Macia varieties, biomass and grain yield WUE decreased in response to low TAW, and irregular and disproportionate rainfall at late planting. In contrast to this, biomass and yield WUE in Ujiba and IsiZulu landraces improved with decreasing rainfall. This 
suggests that PAN8816 can be cropped to increase percentage emergence under low soil water availability, Macia can be cropped at optimal planting to benefit from high yield and HI, whereas Ujiba and IsiZulu landraces are valuable germplasm resources under severe low rainfall conditions. Breeding for improved seedling vigour, yield, HI and low tannin contents in landrace genotypes can potentially assist farmers to improve yield quality and quantity under high rainfall conditions, whilst benefiting from relatively high drought tolerance under low and irregular rainfall. Delayed onset of rainfall and high variability in rainfall during the growing seasons suggest that use of climate forecasts instead of historical data should be recommended for use in selecting planting dates. In this regard, crop models can be useful.

\section{ACKNOWLEDGEMENTS}

The Water Research Commission (WRC) of South Africa is acknowledged for initiating, funding and directing the study through WRC Project No. K5/2274//4 'Determining water use of indigenous grain and legume food crops' (WRC, 2014).

\section{ABBREVIATIONS}

\section{CC: canopy cover}

CCI: chlorophyll content index

HI: harvest index

OPV: open-pollinated variety

SC: stomatal conductance

SSA: sub-Saharan Africa

SWC: soil water content

TAW: total available water

WUE: water use efficiency

\section{REFERENCES}

ADETAYO AO, DAUDA TO, ADETAYO OB, ASIRIBO OE and ISSA F (2008) Rainfall instability difference in the effects of planting dates on growth and yield of maize (Zea mays) in forest savannah eco-climatic zone of Nigeria. Afr. J. Agric. Res. 3 (10) 700-707.

CHAUVIN ND, MULANGU F and PORTO G (2012) Food production and consumption trends in Sub-Saharan Africa: Prospects for the transformation of the agricultural sector. Working Paper 2012-011. United Nations Development Programme. Regional Bureau for Africa, New York.

DELMER DP (2005) Agriculture in the developing world: connecting innovations in plant research to downstream applications. Proc. Natl Acad. Sci. USA 102 15739-15746. https://doi.org/10.1073/ pnas.0505895102

DAFF (Department of Agriculture and Forestry, South Africa) (2010) Sorghum production guidelines. DAFF, Pretoria.

DU PLESSIS J (2008) Sorghum production. Department of Agriculture: Republic of South Africa, and Agricultural Research Council, Pretoria. 1-22.

EJETA G and KNOLL JE (2007) Marker-assisted selection in sorghum. In: Varshney RK and R Tuberosa (ed.) GenomicAssisted Crop Improvement: 2: Genomics Applications in Crops. Springer Netherlands, Dordrecht. 187-205. https://doi. org/10.1007/978-1-4020-6297-1_9

FAO (Food and Agriculture Organization of the United Nations) (1986) Irrigation Water Management. Training Manual No. 3. FAO, Rome.

GREGORY PJ and NORTHCLIFF S (2012) Soil Conditions and Plant Growth. Blackwell Publishing, West Sussex, UK. 288-293.

HENSLEY M, BOTHA JJ, ANDERSON JJ, VAN STADEN PP and DU TOIT A (2000) Optimizing rainfall use efficiency for developing farmers with limited access to irrigation water. WRC Report No. 878/1/00. Water Research Commission, Pretoria.
HUDA AKS, SIVAKURNAR MVK, VIRMANI SM, SEETHARARNA N, SINGH S and SEKARAN JG (1984) Modelling the effect of environmental factors on sorghum growth and development. ICRISAT, Patancheru A.P. 277-287.

JEWITT GWP, WEN HW, KUNZ RP and VAN ROOYEN (2009) Scoping study on water use of crops/trees for biofuels in South Africa. WRC Report No. 1772/1/09. Water Research Commission, Pretoria.

KEATINGE JDH, AIMING Q, KUSMENOGLU I, ELLIS RH, SUMMERFIELD RJ, ERSKINE W and BENIWAL SPS (1995) Defining critical weather events in the phenology of lentil for winter sowing in the west Asian highlands. Agric. For. Meteorol. 74 251-263. https://doi.org/10.1016/0168-1923(94)02186-N

KUSLU Y, SAHIN U, TUNC T and KIZILOGLU FM (2010) Determining water-yield relationship, water use efficiency, seasonal crop and pan coefficients for alfalfa in a semiarid region with high altitude. Bulg. J. Agric. Sci. 16 482-492.

MABHAUDHI T (2012) Drought tolerance and water-use of selected South African landraces of taro (Colocasia esculenta L. Schott) and bambara groundnut (Vigna subterranea $\mathrm{L}$. Verdc). $\mathrm{PhD}$ thesis, University of KwaZulu-Natal, Pietermaritzburg.

MABHAUDHI T and MODI AT (2013) Growth, phenological and yield responses of a bambara groundnut (Vigna subterranea L. Verdc) landrace to imposed water stress: I. Field conditions. S. Afr. J. Plant Soil 30 69-79. https://doi.org/10.1080/02571862.2013.790492

MABHAUDHI T, MODI AT and BELETSE YG (2013) Growth, phenological and yield responses of a bambara groundnut (Vigna subterranea L. Verdc) landrace to imposed water stress: 11. Rain shelter conditions. Water SA 39 191-198. https://doi.org/10.4314/ wsa.v39i2.2

MABHAUDHI T, MODI AT and BELETSE YG (2014) Parameterisation and evaluation of the FAO-AquaCrop model for a South African taro (Colocasia esculenta L. Schott) landrace. Agric. For. Meteorol. 132-139. https://doi.org/10.1016/j.agrformet.2014.03.013

MAMAN N, LYON DJ, MASON S, GALUSHA TD and HIGGINS R (2013) Pearl millet and grain sorghum yield response to water supply in Nebraska. Agron. J. 95 1618-1624. https://doi.org/10.2134/ agronj2003.1618

McMASTER GS and WILHELM WW (1997) Growing degree-days: one equation, two interpretations. Agric. For. Meteorol. 87 291-300. https://doi.org/10.1016/s0168-1923(97)00027-0

MHIZHA T, GEERTS S, VANUYTRECHT E, MAKARAU A and RAES $\mathrm{D}$ (2014) Use of the FAO AquaCrop model in developing sowing guidelines for rainfed maize in Zimbabwe. Water SA 40 233-244. https://doi.org/10.4314/wsa.v40i2.5

RAES D, SITHOLE A, MAKARU A and MILLFORD J (2004) Evaluation of first planting dates recommended by criteria currently used in Zimbabwe. Agric. For. Meteorol. 125 177-185. https://doi. org/10.1016/j.agrformet.2004.05.001

RIDOLFI L, D'ODORICO P, LAIO F, TAMEA S and RODRIGUEZITURBE I (2008) Coupled stochastic dynamics of water table and soil moisture in bare soil conditions. Water Resour. Res. 44 1-11. https:// doi.org/10.1029/2007WR006707

ROCKSTRÖM J and BARRON J (2007) Water productivity in rainfed systems: overview of challenges and analysis of opportunities in water scarcity prone savannahs. Irrig. Sci. 25 299-311. https://doi. org/10.1007/s00271-007-0062-3

SADDAM S, BIBI A, SADAQAT HA and USMAN BF (2014) Comparison of 10 sorghum (Sorghum bicolor L.) genotypes under various water stress regimes. J. Anim. Plant Sci. 24 (5) 1811-1820.

SHENKUTE A, TESFAYE K and ABEGAZ F (2013) Determination of water requirement and crop coefficient for Sorghum (Sorghum bicolor L.) at Melkasa, Ethiopia. STAR J. 2 (3) 16-24.

SMITH B (2006) The farming handbook. University of KwaZulu-Natal Press, Durban. 288-290.

SOIL CLASSIFICATION WORKING GROUP (1991) Soil Classification: A Taxonomic System for South Africa. Soil and Irrigation Research Institute, Department of Agricultural Development, Pretoria.

SWEMMER AM, KNAPP AK and SNYMAN HA (2007) Intraseasonal precipitation patterns and above-ground productivity in three perennial grasslands. J. Ecol. 95 780-788. https://doi. org/10.1111/j.1365-2745.2007.01237.x 
TADROSS MA, HEWITSON BC and USMAN MT (2005) The interannual variability of the onset of the maize growing season over South Africa and Zimbabwe. J. Clim. 18 3356-3372. https://doi. org/10.1175/JCLI3423.1

TOZZI ES, EASLON HM and RICHARDS JH (2013) Interactive effects of water, light and heat stress on photosynthesis in Fremont cottonwood. Plant Cell Environ. 36 (8) 1423-1434. https://doi. org/10.1111/pce. 12070

UDUNGWU BJ and SUMMERFIELD RJ (1985) Comparatory laboratory studies of maize under tropical temperature conditions. East Afr. Agric. For. J. 41 68-74.

VAN HALSEMA GE and VINCENT L (2012) Efficiency and productivity terms for water management: A matter of contextual relativism versus general absolutism. Agric. Water Manage. 108 9-15. https://doi.org/10.1016/j.agwat.2011.05.016

VAN OOSTEROM EJ, CHAPMAN SC, BORRELL AK, BROAD IJ and HAMMER GL (2010) Functional dynamics of the nitrogen balance of sorghum II. Grain filling period. Field Crops Res. 115 29-38. https://doi.org/10.1016/j.fcr.2009.09.019

VOLLENWEIDER P and GÜNTHARDT-GOERG MS (2005) Diagnosis of abiotic and biotic stress factors using the visible symptoms in foliage. Environ. Pollut. 137 455-465. https://doi.org/10.1016/j. envpol.2005.01.032

WRC (Water Research Commission, South Africa) (2014) WRC Knowledge Review 2013/14. Project No. K5/2274//4. Determining water use of indigenous grain and legume food crops. Water Research Commission, Pretoria.

YAMUSA AM, ABU ST, YAHAYA RA, and MUSA IJ (2013) Assessing the planting dates of sorghum in a changing climate at Samaru, Northern Nigeria. Int. J. Biol. Environ. Sci. Trop. 10 (2) 21-26. ZHAO C, FENG Z and CHEN G (2004) Soil water balance simulation of alfalfa (Medicago sativa L.) in the semiarid Chinese Loess Plateau. Agric. Water Manage. 69 101-114. https://doi.org/10.1016/j. agwat.2004.04.006 